\title{
Vector-valued stochastic delay equations-a semigroup approach
}

\author{
Sonja Cox • Mariusz Górajski
}

Received: 7 June 2010 / Accepted: 18 October 2010 / Published online: 3 December 2010

(C) The Author(s) 2010. This article is published with open access at Springerlink.com

Abstract Let $E$ be a type 2 UMD Banach space, $H$ a Hilbert space and let $p \in[1, \infty)$. Consider the following stochastic delay equation in $E$ :

$$
\left\{\begin{array}{l}
d X(t)=A X(t)+C X_{t}+B\left(X(t), X_{t}\right) d W_{H}(t), \quad t>0 \\
X(0)=x_{0} \\
X_{0}=f_{0}
\end{array}\right.
$$

where $A: D(A) \subset E \rightarrow E$ is the generator of a $C_{0}$-semigroup. The operator $C \in \mathcal{L}\left(W^{1, p}(-1,0 ; E), E\right)$ is given by a Riemann-Stieltjes integral, and $B: E \times$ $L^{p}(-1,0 ; E) \rightarrow \gamma(H, E)$ is a Lipschitz function. Moreover $W_{H}$ is an $H$-cylindrical Brownian motion adapted to $\left(\mathscr{F}_{t}\right)_{t \geq 0}$ and $x_{0} \in L^{2}\left(\mathscr{F}_{0}, E\right), f_{0} \in L^{2}\left(\mathcal{F}_{0}, L^{p}(-1,0 ; E)\right)$. We prove that a solution to (SDE) is equivalent to a solution to the corresponding stochastic Cauchy problem, and use this to prove the existence, uniqueness and continuity of a solution to (SDE).

Keywords Stochastic partial differential equations with finite delay $\cdot$ Stochastic Cauchy problem · UMD Banach spaces · Type 2 Banach spaces

Communicated by Markus Haase.

S. Cox $(\bowtie)$

Delft Institute of Applied Mathematics, Delft University of Technology, P.O. Box 5031, 2600 GA

Delft, The Netherlands

e-mail: s.g.cox@tudelft.nl

M. Górajski

Faculty of Mathematics and Computer Science, University of Łódź, Banacha 22, 90-238 Łódź,

Poland

\section{Górajski}

Department of Econometrics, University of Łódź, Rewolucji 1905 r. 41/43, 90-214 Łódź, Poland e-mail: mariuszg@math.uni.lodz.pl 


\section{Introduction}

Let $E$ be a type 2 UMD Banach space and let $H$ be a Hilbert space. Consider the following stochastic delay equation in $E$ :

$$
\left\{\begin{array}{l}
d X(t)=A X(t)+C X_{t}+B\left(X(t), X_{t}\right) d W_{H}(t), \quad t>0 \\
X(0)=x_{0} \\
X_{0}=f_{0}
\end{array}\right.
$$

where for a strongly measurable function $x:[-1, \infty) \rightarrow E$ and $t \geq 0$ we define $x_{t}:[-1,0] \rightarrow E$ by

$$
x_{t}(s):=x(t+s), \quad s \in[-1,0] .
$$

We assume that $A: D(A) \subset E \rightarrow E$ is closed, densely defined and linear, and generates a $C_{0}$-semigroup. Define $\mathcal{E}^{p}(E):=E \times L^{p}(-1,0 ; E)$. We assume that $C \in \mathcal{L}\left(W^{1, p}(-1,0 ; E), E\right)$ for some $p \in[1, \infty)$, and that $B: \mathcal{E}^{p}(E) \rightarrow \gamma(H, E)$ is a Lipschitz function. Here $\gamma(H, E)$ is the space of $\gamma$-radonifying operators from $H$ to $E$, see Sect. 2 below. Moreover, $W_{H}$ is an $H$-cylindrical Brownian motion on a given probability space $\left(\Omega,\left(\mathcal{F}_{t}\right)_{t \geq 0}, \mathcal{F}, \mathbb{P}\right)$. The initial value $\left[x_{0}, f_{0}\right]$ is assumed to be in $L^{2}\left(\mathcal{F}_{0}, \mathcal{E}^{p}(E)\right)$.

Recall that UMD stands for unconditional martingale difference sequences; the class of UMD Banach spaces includes Hilbert spaces and $L^{p}$ spaces for $p \in(1, \infty)$. The type of a Banach space is defined in terms of randomized sequences; see Sect. 4 below. We note that Hilbert spaces have type 2 and $L^{p}$-spaces with $p \in[1, \infty)$ have type $\min \{p, 2\}$.

We follow the semigroup approach to the delay equation as given in the monograph of Batkai and Piazzera [2]. This forces us to assume in addition that $C$ is given by the Riemann-Stieltjes integral

$$
C f:=\int_{-1}^{0} f d \eta,
$$

where $\eta:[-1,0] \rightarrow \mathcal{L}(E)$ is of bounded variation. This defines an element of $\mathcal{L}\left(W^{1, p}(-1,0 ; E), E\right)$ by the Sobolev embedding. (One may allow for more general $C \in \mathcal{L}\left(W^{1, p}(-1,0 ; E), E\right)$; it suffices for $C$ to satisfy the conditions of Theorem 3.26 in [2]. The Riemann-Stieltjes integral is the most important example of such a $C$.)

One can define a closed operator $\mathcal{A}$ on $\mathcal{E}^{p}(E)$ by

$$
\begin{aligned}
D(\mathcal{A}) & =\left\{[x, f] \in D(A) \times W^{1, p}(-1,0 ; E): f(0)=x\right\} ; \\
\mathcal{A} & =\left[\begin{array}{cc}
A & C \\
0 & \frac{d}{d t}
\end{array}\right] .
\end{aligned}
$$

This operator generates a $C_{0}$-semigroup $(\mathcal{T}(t))_{t \geq 0}$ on $\mathcal{E}^{p}(E)$ (see [2], Theorem 3.29) and the stochastic delay equation can be rewritten as a stochastic Cauchy problem in 
$\mathcal{E}^{p}(E)$ given by

$$
\left\{\begin{array}{l}
d Y(t)=\mathcal{A} Y(t) d t+\mathcal{B}(Y(t)) d W_{H}(t), \quad t \geq 0 \\
Y(0)=\left[\begin{array}{l}
x_{0} \\
f_{0}
\end{array}\right],
\end{array}\right.
$$

where $\mathcal{B}(Y(t)):=[B(Y(t)), 0]^{T}$.

The approach we take is to prove existence, uniqueness and continuity of a solution to the stochastic Cauchy problem (SDCP) and then translate these results to corresponding results for the stochastic delay equation (SDE). The monograph by Da Prato and Zabczyk [8] gives an extensive treatment of the stochastic Cauchy problem in Hilbert spaces. The stochastic Cauchy problem in Banach spaces has been considered in the work by Brzeźniak [4] and Van Neerven, Veraar and Weis [24], however, they both consider the case that $\mathcal{A}$ generates an analytic semigroup. Nevertheless their approach is a valuable starting point for studying (SDCP).

Following the approach of the above mentioned authors we consider the following variation of constants formula:

$$
Y(t)=\mathcal{T}(t) Y(0)+\int_{0}^{t} \mathcal{T}(t-s) \mathcal{B}(Y(s)) d W_{H}(s),
$$

where the precise definition of the stochastic integral above and the relevant theory on vector-valued stochastic integrals will be given in Sect. 2. A process satisfying (2) is usually referred to as a mild solution. The existence of a mild solution to (SDCP) is proved by a fixed-point argument (see Sect. 4, Theorem 4.4). Using the factorization method we prove the continuity of a mild solution to (SDCP) (Theorem 4.5). In Sect. 3 we give general conditions under which a mild solution is equivalent to what we call a generalized strong solution of the stochastic Cauchy problem. Finally, Theorem 4.8 states that solutions to (SDCP) and (SDE) are equivalent, which is proved by using the concept of a generalized strong solution. Combining all these results we obtain existence, uniqueness and continuity of a solution to (SDE), see Corollaries 4.10 and 4.11 .

An obvious consequence of our results is that one has the existence of a solution for initial value $f_{0} \in L^{2}\left(\mathcal{F}_{0}, L^{1}(-1,0 ; E)\right)$. The $L^{1}$-norm is a natural choice in population dynamics, see [2, Example 3.16]. The equivalence of solutions to (SDE) and to (SDCP) is useful because the latter can be studied in the framework of the stochastic abstract Cauchy problem; thus answering questions concerning e.g. regularity and invariant measures of the solutions to (SDE) (see Theorem 4.5 and Remark 4.14). We also have that the solution to (SDCP) is a Markov process, whereas the solution to (SDE) is not.

For the theory of stochastic delay equations in the case that $E$ is finite-dimensional we refer to the monographs by Mohammed [16] and Mao [15] and references therein. In particular we wish to mention [5], where equivalence of solutions to the stochastic delay equation and the corresponding abstract Cauchy problem has been shown by Chojnowska-Michalik for the Hilbert space case, i.e. the case that $p=2$ and $E$ is finite-dimensional. Similar results concerning the abstract Cauchy problem arising from delay equations with state space $C([0,1])$ with additive noise are given 
by Van Neerven and Riedle [19]. For a general class of spaces including the $\mathcal{E}^{p}$ spaces the variation of constants formula for finite-dimensional delay equations with additive noise and a bounded delay operator is discussed in Riedle [17]. The latter articles both consider the stochastic convolution as a stochastic integral in a locally convex space. So far there is no suitable interpretation for the stochastic integral of a stochastic process in a locally convex space, hence this approach fails for equations with multiplicative noise.

Stochastic delay equations where $E$ is a Hilbert space and $p=2$ have been considered by Taniguchi, Liu, and Truman [18], Liu [14] and Bierkens, Van Gaans and Verduyn-Lunel [3]. Both [18] and [14] prove existence and uniqueness of solutions to (SDE); in [18] it is assumed that $A$ generates an analytic semigroup, whereas in [14] the noise is assumed to be additive. In [3] the existence of an invariant measure has been studied. Very recently, Crewe [7] has taken it upon himself to prove existence, uniqueness and regularity properties of (SDE) in UMD Banach spaces under the assumption that $A$ generates an analytic semigroup.

\section{Preliminaries: stochastic integration in Banach spaces}

In this section we briefly recall some theory for stochastic integration in Banach spaces as introduced in [23]. Throughout this section let $H, \mathcal{H}$ denote Hilbert spaces and let $F$ denote a Banach space. By $L^{0}(\Omega ; F)$ we denote the complete metric space of strongly measurable functions on $\Omega$ with values in $F$ equipped with the topology of convergence in probability.

To build stochastic integrals of $\mathcal{L}(H, F)$-valued processes we start by considering finite rank adapted step processes, i.e. processes of the form

$$
\Phi(t, \omega)=\sum_{n=1}^{N} 1_{\left(t_{n-1}, t_{n}\right]}(t) \sum_{m=1}^{M} 1_{A_{n m}}(\omega) \sum_{k=1}^{K} h_{k} \otimes x_{n m k},
$$

where $0=t_{0}<t_{1}<\cdots<t_{N}, A_{n m} \in \mathcal{F}_{t_{n-1}}, x_{n m k} \in F$ and $\left(h_{k}\right)_{k \geq 1}$ is an orthonormal system in $H$. If $W_{H}$ is an $H$-cylindrical Brownian motion adapted to $\left(\mathcal{F}_{t}\right)_{t \geq 0}$, then the integral of $\Phi$ with respect to $W_{H}$ is given by:

$$
\int_{0}^{t_{N}} \Phi d W_{H}=\sum_{n=1}^{N} \sum_{m=1}^{M} 1_{A_{n m}} \sum_{k=1}^{K}\left(W_{H}\left(t_{n}\right) h_{k}-W_{H}\left(t_{n-1}\right) h_{k}\right) x_{n m k} .
$$

To extend this to general processes, we need some extra terminology:

Definition 2.1 Let $(\Omega, \mathcal{F}, P)$ be a probability space with filtration $\left(\widetilde{F}_{t}\right)_{t \geq 0}$. A process $\Phi:[0, \infty) \times \Omega \rightarrow \mathcal{L}(H, F)$ is called $H$-strongly measurable if for every $h \in H$ the process $\Phi h$ is strongly measurable. The process is called adapted if $\Phi h$ is adapted for each $h \in H$ and we say that $\Phi$ is scalarly in $L^{q}\left(\Omega ; L^{2}(0, \infty ; H)\right)$ for some $q \in[0, \infty]$ if for all $x^{*} \in F^{*}$ one has $\Phi^{*} x^{*} \in L^{q}\left(\Omega ; L^{2}(0, \infty ; H)\right)$.

The stochastic integral for general $\mathcal{L}(H, F)$-valued processes is defined as follows: 
Definition 2.2 Let $W_{H}$ be an $H$-cylindrical Brownian motion. An $H$-strongly measurable adapted process $\Phi:[0, t] \times \Omega \rightarrow \mathcal{L}(H, F)$ is called stochastically integrable with respect to $W_{H}$ if there exists a sequence of finite rank adapted step processes $\Phi_{n}:[0, t] \times \Omega \rightarrow \mathcal{L}(H, F)$ such that:

(i) for all $h \in H$ and $x^{*} \in F^{*}$ we have $\lim _{n \rightarrow \infty}\left\langle\Phi_{n} h, x^{*}\right\rangle=\left\langle\Phi h, x^{*}\right\rangle$ in measure on $[0, t] \times \Omega$

(ii) there exists a process $X \in L^{0}(\Omega ; C([0, t] ; F))$ such that

$$
\lim _{n \rightarrow \infty} \int_{0}^{\cdot} \Phi_{n} d W_{H}=X \quad \text { in probability. }
$$

The stochastic integral of $\Phi$ is then defined as

$$
\int_{0}^{\cdot} \Phi d W_{H}:=X
$$

A characterization of the processes which are stochastically integrable is obtained by means of the $\gamma$-radonifying norm. Let $\left(\gamma_{j}\right)_{j \geq 1}$ be a sequence of independent standard Gaussian random variables. A bounded operator $R$ from $\mathcal{H}$ to $F$ is called $\gamma$ summing if

$$
\|R\|_{\gamma_{\infty}(\mathcal{H}, F)}^{2}:=\sup _{h} \mathbb{E}\left\|\sum_{j=1}^{k} \gamma_{j} R h_{j}\right\|_{F}^{2}
$$

is finite, where the supremum is taken over all finite orthonormal systems $h=$ $\left(h_{j}\right)_{j=1}^{k}$ in $\mathcal{H}$. It can be shown that $\|\cdot\|_{\gamma_{\infty}(\mathcal{H}, F)}$ is indeed a norm under which the space of $\gamma$-summing operators is complete. We will later take $\mathcal{H}=L^{2}(0, t ; H)$.

The space $\gamma(\mathcal{H}, F)$ of $\gamma$-radonifying operators is defined to be the closure of the finite rank operators under the norm $\|\cdot\|_{\gamma_{\infty}}$; it is a closed subspace of $\gamma_{\infty}(\mathcal{H}, F)$. Thus if $R \in \gamma(\mathcal{H}, F)$ then $\operatorname{range}(R)$ is separable and there exists a separable subspace $\mathcal{H}_{0} \subset \mathcal{H}$ such that $\left.R\right|_{\mathcal{H}_{0}^{\perp}} \equiv 0$.

A celebrated result of Kwapień and Hoffmann-Jørgensen [10, 12] implies that if $F$ does not contain a closed subspace isomorphic to $c_{0}$ then $\gamma(\mathcal{H}, F)=\gamma_{\infty}(\mathcal{H}, F)$. This is the case for the spaces $L^{p}(-1,0 ; F)$ if $p \in[1, \infty)$ and $F$ is a UMD Banach space.

Note also that every $\gamma$-radonifying operator is compact and that the class of $\gamma$ radonifying operators is a left- and right ideal in the set of bounded operators:

$$
\|S R T\|_{\gamma\left(\mathcal{H}_{1}, F_{2}\right)} \leq\|S\|_{\mathcal{L}\left(F_{1}, F_{2}\right)}\|R\|_{\gamma\left(\mathcal{H}_{2}, F_{1}\right)}\|T\|_{\mathcal{L}\left(\mathcal{H}_{1}, \mathcal{H}_{2}\right)},
$$

where $\mathcal{H}_{1}, \mathcal{H}_{2}$ are Hilbert spaces and $F_{1}, F_{2}$ are Banach spaces.

In what follows we will use the notation $A \lesssim_{p} B$ to express the fact that there exists a constant $C>0$, depending on $p$, such that $A \leq C B$. We write $A{\sim_{p}}_{B}$ if $A \lesssim_{p} B \lesssim_{p} A$.

Theorem 5.9 and Theorem 5.12 in [23] state the relation between the $\gamma$-radonifying norm and the stochastically integrable processes (see also [6] for relation (3)). We summarize these results as follows: 
Theorem 2.3 Let $F$ be a UMD space. For an $H$-strongly measurable adapted process $\Phi:[0, t] \times \Omega \rightarrow \mathcal{L}(H, F)$ belonging to $L^{0}\left(\Omega ; L^{2}(0, t ; H)\right)$ scalarly, the following are equivalent:

(i) $\Phi$ is stochastically integrable;

(ii) there exists a process $\eta \in L^{0}(\Omega ; C([0, t] ; F))$ such that for all $x^{*} \in F^{*}$ we have

$$
\left\langle\eta, x^{*}\right\rangle=\int_{0}^{\cdot} \Phi^{*}(s) x^{*} d W_{H}(s) \quad \text { a.s. }
$$

(iii) $\Phi$ represents an element $R_{\Phi} \in L^{0}\left(\Omega ; \gamma\left(L^{2}(0, t ; H), F\right)\right)$ which is defined as follows:

$$
R_{\Phi}(\omega) f:=\int_{0}^{t} \Phi(s, \omega) f(s) d s
$$

$\left(f \in L^{2}(0, t ; H)\right)$.

In this situation one has $\eta=\int_{0}^{\cdot} \Phi d W_{H}$ and for all $p \in(0, \infty)$

$$
\mathbb{E} \sup _{0 \leq s \leq t}\left\|\int_{0}^{s} \Phi(u) d W_{H}(u)\right\|_{F}^{p} \bar{\sim}_{p} \mathbb{E}\left\|R_{\Phi}\right\|_{\gamma\left(L^{2}(0, t ; H), F\right)}^{p} .
$$

Remark 2.4 If $\Phi$ is $H$-strongly measurable and $R_{\Phi} \in \gamma\left(L^{2}(0, t ; H), F\right)$ a.s. then by [23, Lemma $2.5,2.7$ and Remark 2.8] one automatically obtains that $R_{\Phi} \in$ $L^{0}\left(\Omega ; \gamma\left(L^{2}(0, t ; H) ; F\right)\right)$. Thus in this situation one may assume without loss of generality that $H$ and $F$ are separable.

From now on we shall simply write $\|\Phi\|_{\gamma(0, t ; H, F)}$ to denote the $\gamma\left(L^{2}(0, t ; H), F\right)$ norm of the operator $R_{\Phi}$ associated with $\Phi$.

Remark 2.5 One checks that if (iii) in the theorem above holds, then $\Phi$ must be scalarly in $L^{0}\left(\Omega ; L^{2}(0, t ; H)\right)$. Moreover, the implication (i) $\Rightarrow$ (ii) holds for arbitrary Banach spaces. This follows from the Burkholder-Davis-Gundy inequalities (see the proof of Theorem 3.6 in [23]).

For $1<p<\infty$ one has that if $F$ is a UMD space then so is $\mathcal{E}^{p}(F)$. However, $L^{1}$ is not a UMD space so neither is $\mathcal{E}^{1}(-1,0 ; F)$. Fortunately, $L^{1}$ does have the (weaker) decoupling property as introduced by Kwapień and Woyczyński in [13]. If $F$ has the decoupling property then $\mathcal{E}^{p}(F)$ is Banach space satisfying the decoupling inequality (by a Fubini argument, see [6]). It was proved in [6] that for spaces with the decoupling property implication (iii) $\Rightarrow$ (i) remains valid. The two-sided estimate as given in (3) need not hold in such spaces, but it is shown in [6] that in spaces with the decoupling property the following one-sided estimate holds for all $p \in(0, \infty)$ :

$$
\mathbb{E} \sup _{0 \leq s \leq t}\left\|\int_{0}^{s} \Phi(u) d W_{H}(u)\right\|_{F}^{p} \lesssim_{p} \mathbb{E}\|\Phi\|_{\gamma(0, t ; H, F)}^{p} .
$$

Note that in particular the integral process $t \mapsto \int_{0}^{t} \Phi(s) d W_{H}(s)$ is continuous. 
For $\gamma$-radonifying operators with values in an $L^{p}$-space we have the following isomorphism (see [24], Proposition 2.6):

Lemma 2.6 Let $(S, \mathcal{S}, \mu)$ be a $\sigma$-finite measure space, let $\mathcal{H}$ be a Hilbert space and let $p \in[1, \infty)$. Then the mapping $U: L^{p}(S ; \gamma(\mathcal{H}, F)) \rightarrow \mathcal{L}\left(\mathcal{H}, L^{p}(S ; F)\right)$ defined by

$$
((U f) h)(\xi):=f(\xi) h, \quad \xi \in S, h \in \mathcal{H},
$$

defines an isomorphism $U$ of $L^{p}(S ; \gamma(\mathcal{H}, F))$ onto $\gamma\left(\mathcal{H}, L^{p}(S ; F)\right)$.

The following stochastic Fubini theorem is based on [20, Theorem 3.5].

Lemma 2.7 Let $(S, \mathcal{S}, \mu)$ be a $\sigma$-finite measure space and let $F$ be a Banach space satisfying the decoupling property. Let $\Phi: S \times[0, t] \times \Omega \rightarrow \mathcal{L}(H, F)$ and for $s \in S$ define $\Phi_{s}:[0, t] \times \Omega \rightarrow \mathcal{L}(H, F)$ by $\Phi_{s}(u, \omega)=\Phi(s, u, \omega)$. Assume the following is satisfied:

(i) $\Phi$ is $H$-strongly measurable;

(ii) For all $s \in S$ and all $h \in H$ the section $\Phi_{s} h$ is progressive;

(iii) For almost all $u \in[0, t]$ and almost all $\omega \in \Omega$ one has $\Phi(\cdot, u, \omega) h \in L^{1}(S ; F)$ for all $h \in H$ and the operator $\int_{S} \Phi d \mu: H \rightarrow F$ defined by $\int_{S} \Phi d \mu h:=$ $\int_{S}$ Фhd $\mu$ is in $\mathcal{L}(H, F)$;

(iv) The process $u \mapsto \int_{S} \Phi(s, u) d \mu(s)$ represents an element of $\gamma(0, t ; H, F)$ a.s.;

(v) The function $s \mapsto \Phi_{s}$ represents an element of $L^{1}(S ; \gamma(0, t ; H, F))$ a.s.

Then the function $s \mapsto \int_{0}^{t} \Phi(s, u) d W_{H}(u)$ belongs to $L^{1}(S ; F)$ a.s. and

$$
\int_{S} \int_{0}^{t} \Phi d W_{H} d \mu=\int_{0}^{t} \int_{S} \Phi d \mu d W_{H} \quad \text { a.s. }
$$

Proof Due to condition (v) and the Fubini isomorphism in Lemma 2.6 one has that $\Phi$ represents an element of $\gamma\left(0, t ; H, L^{1}(S ; F)\right)$ a.s. As $\Phi$ is assumed to be $H$-strongly measurable we may assume $H$ and $F$ to be separable by Remark 2.4. This implies that $\Phi^{*} x^{*}$ is strongly measurable for all $x^{*} \in F^{*}$ by Pettis's measurability theorem, and that $\Phi_{s}^{*} x^{*}$ is progressive for all $x^{*} \in F^{*}$, all $s \in S$ and all $h \in H$.

Moreover, because $\Phi$ represents an element of $\gamma\left(0, t ; H, L^{1}(S ; F)\right)$ a.s., the process $\Psi:[0, t] \times \Omega \rightarrow \mathcal{L}\left(H, L^{1}(S ; F)\right)$ defined by

$$
\Psi(u, \omega)(s):=\Phi(s, u, \omega)
$$

is stochastically integrable, and by arguments similar to those in the proof of [20, Theorem 3.5] it follows that

$$
\int_{0}^{t} \Phi(s, u) d W_{H}(u)=\left(\int_{0}^{t} \Psi(u) d W_{H}(u)\right)(s) \quad \text { a.s. for almost all } s \in S .
$$

This proves that the integral with respect to $\mu$ on the left-hand side of (5) is welldefined. 
Condition (iii) implies that the process in condition (iv) is well-defined, and this condition in combination with Theorem 2.3 and Remark 2.5 implies that the stochastic integral on the right-hand side of (5) is well-defined.

Fix $x^{*} \in F^{*}$, then $\Phi^{*} x^{*}: S \times[0, t] \times \Omega \rightarrow H$ satisfies conditions (i)-(iii) of [20, Theorem 3.5] and hence by that theorem we have:

$$
\int_{S} \int_{0}^{t} \Phi^{*} x^{*} d W_{H} d \mu=\int_{0}^{t} \int_{S} \Phi^{*} x^{*} d \mu d W_{H} \quad \text { a.s. }
$$

Although the null-set on which the above fails may depend on $x^{*}$, this suffices due to the fact that $F^{*}$ is weak ${ }^{*}$-separable.

In the next section we will need the following lemma which shows that as in the case of the Bochner integral, a closed operator can be taken out of a stochastic integral.

Lemma 2.8 Let $F$ be a Banach space satisfying the decoupling property and let $A: D(A) \subset F \rightarrow F$ be a closed, densely defined operator. Suppose $\Phi:[0, t] \times \Omega \rightarrow$ $\mathcal{L}(H, F)$ is an $H$-strongly measurable adapted process that represents an element of $\gamma(0, t ; H, F)$ a.s. Suppose that one has $\Phi(s) h \in D(A)$ for all $s \in(0, t)$ and all $h \in H$ a.s., where the null sets are independent of $h$. Suppose moreover that $A \Phi$ is again an $H$-strongly measurable adapted process that represents an element of $\gamma(0, t ; H, F)$ a.s. Then $\int_{0}^{t} \Phi d W_{H} \in D(A)$ a.s. and

$$
A \int_{0}^{t} \Phi d W_{H}=\int_{0}^{t} A \Phi d W_{H} \quad \text { a.s. }
$$

Proof Define random variables $\eta:=\int_{0}^{t} \Phi d W_{H}$ and $\zeta:=\int_{0}^{t} A \Phi d W_{H}$ and observe that by implication (iii) $\Longrightarrow$ (ii) in Theorem 2.3, which holds for Banach spaces with decoupling property, one has that for all $x^{*} \in F^{*}$ :

$$
\begin{aligned}
& \left\langle\eta, x^{*}\right\rangle=\int_{0}^{t} \Phi^{*}(s) x^{*} d W_{H}(s) \quad \text { a.s., } \\
& \left\langle\zeta, x^{*}\right\rangle=\int_{0}^{t}(A \Phi(s))^{*} x^{*} d W_{H}(s) \quad \text { a.s. }
\end{aligned}
$$

In particular for $x^{*} \in D\left(A^{*}\right)$ one has $(A \Phi(s))^{*} x^{*}=\Phi^{*}(s) A^{*} x^{*}$, and thus for such $x^{*}$ one has:

$$
\left\langle(\eta, \zeta),\left(-A x^{*}, x^{*}\right)\right\rangle=\left\langle\eta,-A^{*} x^{*}\right\rangle+\left\langle\zeta, x^{*}\right\rangle=0 \quad \text { a.s. }
$$

Note that the null-set on which the equation above fails to hold may depend on $x^{*}$. However, as $\Phi$ and $A \Phi$ are assumed to be $H$-strongly measurable and in $\gamma(0, t ; H, F)$ a.s. we may assume $F$ to be separable by Remark 2.4. Hence $(F \times F) / \mathcal{G}(A)$ is separable, where $\mathcal{G}(A)$ is the graph of $A$, and thus by Hahn-Banach there exists a countable subset of $((F \times F) / \mathcal{G}(A))^{*}=\mathcal{G}(A)^{\perp}$ that separates the points of $(F \times F) / \mathcal{G}(A)$. 
Moreover, one checks that if $\left(x_{1}^{*}, x_{2}^{*}\right) \in \mathcal{G}(A)^{\perp}$ then $x_{2}^{*} \in D\left(A^{*}\right)$ and $x_{1}^{*}=$ $-A^{*} x_{2}^{*}$. Thus there exists a sequence $\left(-A x_{n}^{*}, x_{n}^{*}\right)_{n \in \mathbb{N}}$ that separates points in $(F \times$ $F) / \mathcal{G}(A)$. As (6) holds for arbitrary $x^{*} \in D\left(A^{*}\right)$, it holds simultaneously for all $x_{n}^{*}$, on a set of measure one. Therefore $(\eta, \zeta) \in \mathcal{G}(A)$, i.e. $\eta \in D(A)$ and $A \eta=\zeta$ a.s.

\section{The stochastic Cauchy problem}

In the introduction we mentioned that the stochastic delay equation (SDE) can be rewritten as a stochastic Cauchy problem. In this section we briefly consider the stochastic Cauchy problem in general. Let $F$ be a Banach space with the decoupling property and $H$ a Hilbert space, and let $A: D(A) \subset F \rightarrow F$ be the generator of a $C_{0}$-semigroup $(T(t))_{t \geq 0}$ on $F$. Let $W_{H}$ be an $H$-cylindrical Brownian motion and let $B: F \rightarrow \mathcal{L}(H, F)$ be continuous (where $\mathcal{L}(H, F)$ is endowed with the strong operator topology). We consider the following problem:

$$
\left\{\begin{array}{l}
d Y(t)=A Y(t) d t+B(Y(t)) d W_{H}(t), \quad t \geq 0 \\
Y(0)=Y_{0}
\end{array}\right.
$$

Definition 3.1 An $H$-strongly measurable adapted process $Y$ is called a generalized strong solution to (SCP) if $Y$ is a.s. locally Bochner integrable and for all $t>0$ :

(i) $\int_{0}^{t} Y(s) d s \in D(A)$ a.s.,

(ii) $B(Y)$ is stochastically integrable on $[0, t]$,

and

$$
Y(t)-Y_{0}=A \int_{0}^{t} Y(s) d s+\int_{0}^{t} B(Y(s)) d W_{H}(s) \quad \text { a.s. }
$$

We use the term 'generalized strong solution' to distinguish this solution concept from the conventional definition of a 'strong solution', which concerns a process satisfying $Y(t) \in D(A)$ a.s. for all $t \geq 0$ (see [8]). This assumption is not suitable for our situation, see Remark 4.12 below.

Theorem 3.2 Let $Y$ be an $F$-valued $H$-strongly measurable adapted process. For $t \geq 0$ define $\int_{0}^{t} T(s) B(Y(u)) d s \in \mathcal{L}(H, F)$ by

$$
\left(\int_{0}^{t} T(s) B(Y(u)) d s\right) h:=\int_{0}^{t} T(s) B(Y(u)) h d s .
$$

Assume that for all $t>0$ the following processes are in $\gamma(0, t ; H, F)$ a.s.:

(a) $B(Y)$;

(b) $u \mapsto T(t-u) B(Y(u))$;

(c) $u \mapsto \int_{0}^{t-u} T(s) B(Y(u)) d s$;

and that for all $t>0$

$$
\int_{0}^{t}\|T(s-\cdot) B(Y(\cdot))\|_{\gamma(0, s, H ; F)} d s<\infty .
$$


Then $Y$ is a generalized strong solution to (SCP) if and only if $Y$ satisfies, for all $t \geq 0$,

$$
Y(t)=T(t) Y_{0}+\int_{0}^{t} T(t-s) B(Y(s)) d W_{H}(s) \quad \text { a.s. }
$$

Remark 3.3 (1) If $Y$ is strongly measurable and adapted then the processes in (a), (b) and (c) are $H$-strongly measurable and adapted.

(2) If $B: F \rightarrow \gamma(H, F)$ then for all $u \in[0, t]$ almost all paths $s \mapsto T(s) B(Y(u))$ are locally Bochner integrable in $\gamma(H, F)$ because $B(Y(u))$ is the limit of finite-rank operators in $\gamma(H, F)$.

(3) If $F$ is a UMD space and $(T(s))_{0 \leq s \leq t}$ is $\gamma$-bounded for all $t>0$ then (b) and (c) follow from (a). For definition and details on $\gamma$-boundedness see [24], analytic semigroups are a typical example of $\gamma$-bounded semigroups.

Proof of Theorem 3.2 Step 1. We apply Lemmas 2.7 and 2.8 to obtain the key equations for the proof of Theorem 3.2, equations (11) and (12) below. As every adapted and measurable process with values in Polish space has a progressive version we may assume that $Y$ is progressive. Consider the following process:

$$
\Phi:[0, t] \times[0, t] \times \Omega \rightarrow \mathcal{L}(H, F) ; \quad \Phi(s, u, \omega):=1_{u \leq s \leq t} T(t-s) B(Y(u)) .
$$

Because $Y$ is strongly measurable, and because $B: F \rightarrow \mathcal{L}(H, F)$ is continuous with respect to the strong operator topology and the semigroup $T(s)$ is strongly continuous it follows that $\Phi$ is $H$-strongly measurable. Similarly, it follows from the fact that $Y$ is progressive that for all $s \in[0, t]$ and all $h \in H$ the section $\Phi_{s} h$ is progressive. Thus conditions (i) and (ii) of Lemma 2.7 are satisfied. One easily checks that condition (iii) of Lemma 2.7 is satisfied by $\Phi$. Condition (iv) in Lemma 2.7 follows from assumption (c). Condition (v) in Lemma 2.7 follows from the definition of $\gamma(0, t ; H, F)$, assumption (a) and the exponential boundedness of the semigroup: let $\left(h_{k}\right)_{k=1}^{n}$ be an arbitrary orthonormal sequence in $L^{2}(0, t ; H)$, then

$$
\begin{aligned}
& \int_{0}^{t}\left(\mathbb{E}\left\|\sum_{k=1}^{n} \gamma_{k} \int_{0}^{t} T(t-s) B(Y(u)) h_{k}(u) 1_{[0, s]}(u) d u\right\|_{F}^{2}\right)^{\frac{1}{2}} d s \\
& \quad \leq \int_{0}^{t}\|T(t-s)\|_{\mathcal{L}(F)}\left(\mathbb{E}\left\|\sum_{k=1}^{n} \gamma_{k} \int_{0}^{t} B(Y(u)) h_{k}(u) 1_{[0, s]}(u) d u\right\|_{F}^{2}\right)^{\frac{1}{2}} d s \\
& \quad \leq M_{t} \int_{0}^{t}\left\|B(Y) 1_{[0, s]}\right\|_{\gamma(0, t ; H, F)} d s \leq t M_{t}\|B(Y)\|_{\gamma(0, t ; H, F)}<\infty,
\end{aligned}
$$

where $M_{t}:=\sup _{0 \leq s \leq t}\|T(s)\|_{\mathcal{L}(F)}$ and we used the domination principle for Gaussian random variables to see that $\left\|B(Y) 1_{[0, s]}\right\|_{\gamma(0, t ; H, F)} \leq\|B(Y)\|_{\gamma(0, t ; H, F)}$. Thus $\Phi$ satisfies all the conditions of the stochastic Fubini Lemma and we obtain:

$$
\int_{0}^{t} T(t-s) \int_{0}^{s} B(Y(u)) d W_{H}(u) d s=\int_{0}^{t} \int_{u}^{t} T(t-s) B(Y(u)) d s d W_{H}(u) \quad \text { a.s. }
$$


Observe that for all $h \in H$ one has $\int_{u}^{t} T(t-s) B(Y(u)) h d s \in D(A)$. Hence by assumptions (a) and (b) we can apply Lemma 2.8 to obtain that the stochastic integral on the right-hand side of (9) above is in $D(A)$ a.s., and we have:

$$
\begin{aligned}
A \int_{0}^{t} \int_{u}^{t} T(t-s) B(Y(u)) d s d W_{H}(u) & =\int_{0}^{t} A \int_{0}^{t-u} T(s) B(Y(u)) d s d W_{H}(u) \\
& =\int_{0}^{t}(T(t-u)-I) B(Y(u)) d W_{H}(u) \text { a.s. }
\end{aligned}
$$

Combining (9) and (10) we obtain:

$$
A \int_{0}^{t} T(t-s) \int_{0}^{s} B(Y(u)) d W_{H}(u) d s=\int_{0}^{t}(T(t-u)-I) B(Y(u)) d W_{H}(u) \quad \text { a.s. }
$$

Similarly using assumption (7) one can prove that for $0 \leq s \leq t$ the stochastic integrals in the equation below are well-defined and one has the following identity:

$$
A \int_{0}^{t} \int_{0}^{s} T(s-u) B(Y(u)) d W_{H}(u) d s=\int_{0}^{t}(T(t-u)-I) B(Y(u)) d W_{H}(u) .
$$

Step 2. Assume $Y$ is a generalized strong solution to (SCP), we prove that (8) holds. By (11) and by the definition of a generalized strong solution we have:

$$
\begin{aligned}
Y(t) & -Y_{0}-A \int_{0}^{t} Y(s) d s \\
= & \int_{0}^{t} B(Y(s)) d W_{H}(s) \\
= & \int_{0}^{t} T(t-s) B(Y(s)) d W_{H}(s)-A \int_{0}^{t} T(t-s) \int_{0}^{s} B(Y(u)) d W_{H}(u) d s .
\end{aligned}
$$

Let us consider the final term above without the $A$. By assumption and by Fubini's theorem one has:

$$
\begin{aligned}
& \int_{0}^{t} T(t-s) \int_{0}^{s} B(Y(u)) d W_{H}(u) d s \\
& \quad=\int_{0}^{t} T(t-s)\left[Y(s)-Y_{0}-A \int_{0}^{s} Y(u) d u\right] d s \\
& \quad=\int_{0}^{t} T(t-s) Y(s) d s-\int_{0}^{t} T(t-s) Y_{0} d s-A \int_{0}^{t} \int_{u}^{t} T(t-s) Y(u) d s d u \\
& =-\int_{0}^{t} T(t-s) Y_{0} d s+\int_{0}^{t} Y(s) d s,
\end{aligned}
$$


which, when substituted to the earlier equation, gives:

$$
\begin{aligned}
Y(t) & -Y_{0}-A \int_{0}^{t} Y(s) d s \\
& =\int_{0}^{t} T(t-s) B(Y(s)) d W_{H}(s)+T(t) Y_{0}-Y_{0}-A \int_{0}^{t} Y(s) d s .
\end{aligned}
$$

On the other hand, if $Y$ satisfies (8), then $\int_{0}^{t} Y(s) d s$ exists and is in $D(A)$ a.s. by (12), and therefore using this equation we obtain:

$$
\begin{aligned}
A \int_{0}^{t} Y(s) d s & =A \int_{0}^{t} T(s) Y_{0} d s+A \int_{0}^{t} \int_{0}^{s} T(s-u) B(Y(u)) d W_{H}(u) d s \\
& =T(t) Y_{0}-Y_{0}+\int_{0}^{t}[T(t-u)-1] B(Y(u)) d W_{H}(u) \\
& =Y(t)-Y_{0}-\int_{0}^{t} B(Y(u)) d W_{H}(u) .
\end{aligned}
$$

Continuity of a process satisfying (8) can be proved by means of the factorization method as introduced in Sect. 2 of [9]. We give the proof below; it is a straightforward adaptation of the proof of Theorem 3.3 in [25].

Theorem 3.4 Let $(T(t))_{t \geq 0}$ be a semigroup on a Banach space $F$ with the decoupling property. Let $Z:[0, t] \times \Omega \rightarrow \mathcal{L}(H, F)$ be an $H$-strongly measurable adapted process. Suppose that there exists $\alpha, p>0, \frac{1}{p}<\alpha<\frac{1}{2}$ and $M>0$ such that

$$
\sup _{0 \leq s \leq t}\left\|u \mapsto(s-u)^{-\alpha} T(s-u) Z(u)\right\|_{L^{p}(\Omega ; \gamma(0, s, H ; F))} \leq M .
$$

Then the process

$$
s \mapsto \int_{0}^{s} T(s-u) Z(u) d W_{H}(u)
$$

is well-defined and has a version with continuous paths. Moreover we have

$$
\mathbb{E} \sup _{0 \leq s \leq t}\left\|\int_{0}^{s} T(s-u) Z(u) d W_{H}(u)\right\|_{F}^{p}<\infty .
$$

Before giving the proof of this theorem we mention the following corollary:

Corollary 3.5 Consider the stochastic Cauchy problem (SCP) set in a Banach space $F$ that satisfies the decoupling property. The process $Y:[0, t] \times \Omega \rightarrow F$ satisfying the variation of constants formula (8) belongs to $L^{p}(\Omega ; C([0, t] ; F))$ if there exists $\alpha, p>0, \frac{1}{p}<\alpha<\frac{1}{2}$ such that

$$
\sup _{0 \leq s \leq t}\left\|u \mapsto(s-u)^{-\alpha} T(s-u) Y(u)\right\|_{L^{p}(\Omega ; \gamma(0, s, H ; F))}<\infty .
$$


Proof of Theorem 3.4 By assumption (13) and Theorem 2.3 it follows that for all $s \in[0, t]$ we can define

$$
\Psi_{1}(s):=\int_{0}^{s}(s-u)^{-\alpha} T(s-u) Z(u) d W_{H}(u) .
$$

By Proposition A.1 in [24] the process $\Phi_{1}$ has a version which is adapted and strongly measurable. Moreover, by assumption and inequality (4) one has, for all $s \in[0, t]$,

$$
\mathbb{E}\left\|\Psi_{1}(s)\right\|_{F}^{p} \leq M,
$$

whence $\Psi_{1} \in L^{p}\left(0, t ; L^{p}(\Omega ; F)\right)$, and thus, by Fubini, $\Psi_{1} \in L^{p}\left(\Omega ; L^{p}(0, t ; F)\right)$. Let $\Omega_{0} \subset \Omega$ denote the set on which $\Psi_{1} \in L^{p}(0, t ; F)$; we have $\mathbb{P}\left(\Omega_{0}\right)=1$.

By the domination principle for Gaussian random variables (see also [21, Corollary 4.4]) it follows that for all $s \in[0, t]$ one has, almost surely,

$$
\begin{aligned}
\| u & \mapsto T(s-u) Z(u, \omega) \|_{\gamma(0, s, H ; F)} \\
& \leq\left\|u \mapsto t^{\alpha}(s-u)^{-\alpha} T(s-u) Z(u, \omega)\right\|_{\gamma(0, s, H ; F)} \text { a.s. }
\end{aligned}
$$

Thus by assumption we can define, for all $s \in[0, t]$,

$$
\Psi_{2}(s):=\int_{0}^{s} T(s-u) Z(u) d W_{H}(u)
$$

which again has a version that is adapted and strongly measurable.

It is proved in [9] that one may define a bounded operator $R_{\alpha}: L^{p}(0, t ; F) \rightarrow$ $C([0, t] ; F)$ by setting

$$
\left(R_{\alpha} f\right)(s):=\int_{0}^{s}(s-u)^{\alpha-1} T(s-u) f(u) d u .
$$

Thus it remains to show that for almost all $\omega \in \Omega_{0}$ one has that for all $s \in[0, t]$ that

$$
\Psi_{2}(s)=\frac{\sin \pi \alpha}{\pi}\left(R_{\alpha} \Psi_{1}\right)(s),
$$

i.e. that for all $x^{*} \in F^{*}$ one has

$$
\left\langle\Psi_{2}(s), x^{*}\right\rangle=\frac{\sin \pi \alpha}{\pi} \int_{0}^{s}(s-u)^{\alpha-1}\left\langle T(s-u) \Psi_{1}(u), x^{*}\right\rangle d u \quad \text { a.s. }
$$

This follows from a Fubini argument, see [20, Theorem 3.5] and [9]. The conditions necessary to apply the Fubini Theorem follow from the assumption (13). By (15) and (14) one has

$$
\mathbb{E} \sup _{0 \leq s \leq t}\left\|\Psi_{2}(s)\right\|_{F}^{p} \leq C \mathbb{E} \int_{0}^{t}\left\|\Psi_{1}(s)\right\|_{F}^{p} d s \leq t C M,
$$

where $C$ is independent of $Z$. Thus the final estimate follows. 


\section{The stochastic delay equation}

\subsection{The variation of constants formula}

We now turn to the stochastic delay equation (SDE) as presented in the introduction and the related stochastic Cauchy problem (SDCP) on p. 391. Recall that we assumed that (SDE) is set in a type 2 UMD Banach space $E$ and that the related Cauchy problem is set in $\mathcal{E}^{p}(E)=E \times L^{p}(-1,0 ; E)$ for some $p \in[1, \infty)$. (The results in this article are also valid if $E$ is a type 2 Banach space with the decoupling property but we do not know of any such spaces that are not in fact UMD spaces.)

Recall that a Banach space $F$ is said to have type $p \in[1,2]$ if there exists a constant $C \geq 0$ such that for all finite choices $x_{1}, \ldots, x_{k} \in F$ we have

$$
\left(\mathbb{E}\left\|\sum_{j=1}^{k} \gamma_{j} x_{j}\right\|_{F}^{2}\right)^{\frac{1}{2}} \leq C\left(\sum_{j=1}^{k}\left\|x_{j}\right\|_{F}^{p}\right)^{\frac{1}{p}},
$$

where $\left(\gamma_{j}\right)_{j \geq 1}$ is a sequence of independent standard Gaussians. Hilbert spaces have type 2 and $L^{p}$-spaces with $p \in[1, \infty)$ have type $\min \{p, 2\}$. We refer to [1] for more information, for our purposes we only need that in Banach spaces with type 2 the following embedding holds, see p. 1460 in [23]:

$$
L^{2}(0, t ; \gamma(H, F)) \hookrightarrow \gamma(0, t ; H, F) .
$$

Let $(\mathcal{T}(t))_{t \geq 0}$ denote the semigroup generated by $\mathcal{A}$, where $\mathcal{A}$ is the operator in (SDCP) defined by (1) in the introduction. We define the projections $\pi_{1}: \mathcal{E}^{p}(E) \rightarrow E$ and $\pi_{2}: \mathcal{E}^{p}(E) \rightarrow L^{p}(-1,0 ; E)$ as follows:

$$
\pi_{1}\left[\begin{array}{l}
x \\
f
\end{array}\right]=x ; \quad \pi_{2}\left[\begin{array}{l}
x \\
f
\end{array}\right]=f .
$$

The following property of $(\mathcal{T}(t))_{t \geq 0}$ is intuitively obvious and useful in the following:

$$
\left(\pi_{2} \mathcal{T}(t)\left[\begin{array}{l}
x \\
f
\end{array}\right]\right)(u)=\pi_{1} \mathcal{T}(t+u)\left[\begin{array}{l}
x \\
f
\end{array}\right]
$$

for $f \in \mathcal{E}^{p}(E), u \in[-1,0], t>-u$ (for a proof see [2], Proposition 3.11).

The proof of the following lemma is straightforward and thus left to the reader:

Lemma 4.1 Let $t>0, p \in[1, \infty)$ and $x \in L^{p}(-1, t ; E)$. Then the function $y$ : $[0, t] \rightarrow L^{p}(-1,0 ; E), y(s):=x_{s}$ is (Bochner) integrable and

$$
\begin{aligned}
& \int_{0}^{t} y(s) d s \in W^{1, p}(-1,0 ; E) ; \\
& \left(\int_{0}^{t} y(s) d s\right)(u)=\int_{0}^{t} x(s+u) d s \quad \text { a.s.; } \quad\left(\int_{0}^{t} y(s) d s\right)^{\prime}=y(t)-y(0) \text { a.s. }
\end{aligned}
$$


Generalized strong solutions to (SDCP) are equivalent to mild solutions:

Theorem 4.2 Let $E$ be a type 2 UMD Banach space and let $p \in[1, \infty)$. Consider (SDCP); i.e. let $\mathcal{A}$ defined by (1) be the generator of the $C_{0}$-semigroup $(\mathcal{T}(t))_{t \geq 0}$ on $\mathcal{E}^{p}(E)=E \times L^{p}(-1,0 ; E)$. Let $\mathcal{B}: \mathcal{E}^{p}(E) \rightarrow \gamma\left(H, \mathcal{E}^{p}(E)\right)$ be given by $\mathcal{B}\left([x, f]^{T}\right)=[B([x, f]), 0]^{T}$, where $B: \mathcal{E}^{p}(E) \rightarrow \gamma(H, E)$ is Lipschitz continuous. Finally, let $W_{H}$ be an $H$-cylindrical Brownian motion adapted to $\left(\mathcal{F}_{S}\right)_{s \geq 0}$.

Let $Y:[0, \infty) \times \Omega \rightarrow \mathcal{E}^{p}(E)$ be a strongly measurable, adapted process satisfying

$$
\int_{0}^{t}\|Y(s)\|_{\mathcal{E}^{p}(E)}^{2} d s<\infty \quad \text { a.s. for all } t>0
$$

Then $Y$ is a generalized strong solution to (SDCP) if and only if $Y$ is a solution to:

$$
Y(t)=\mathcal{T}(t)\left[\begin{array}{l}
x_{0} \\
f_{0}
\end{array}\right]+\int_{0}^{t} \mathcal{T}(t-s) \mathcal{B}(Y(s)) d W_{H}(s) \quad \text { a.s. for all } t \geq 0 .
$$

Proof We apply Theorem 3.2 to obtain the above assertion, for which we need to check condition (7) and that the processes given by (a), (b) and (c) in that theorem are elements of $\gamma\left(0, t ; H, \mathcal{E}^{p}(E)\right)$ a.s. for all $t>0$. Let $t>0$ be fixed.

Process (a) in Theorem 3.2. By the embedding (16) and the Lipschitz-continuity of $\mathcal{B}$ we have:

$$
\begin{aligned}
\|s \mapsto \mathcal{B}(Y(s))\|_{\gamma(0, t ; H, \mathcal{E} p(E))} & =\|s \mapsto B(Y(s))\|_{\gamma(0, t ; H, E)} \\
& \lesssim\|s \mapsto B(Y(s))\|_{L^{2}(0, t ; \gamma(H, E))} \\
& \lesssim t^{\frac{1}{2}}\|B(0)\|_{\gamma(H, E)}+K\|Y\|_{L^{2}\left(0, t ; \mathcal{E}^{p}(E)\right)},
\end{aligned}
$$

where $K$ is the Lipschitz-constant of $B$.

Process (b) in Theorem 3.2. By Lemma 2.6 and embedding (16) we have:

$$
\begin{aligned}
\| u & \mapsto \mathcal{T}(t-u) \mathcal{B}(Y(u)) \|_{\gamma\left(0, t ; H, \mathcal{E}^{p}(E)\right)} \\
\lesssim_{p}\left\|u \mapsto \pi_{1} \mathcal{T}(t-u) \mathcal{B}(Y(u))\right\|_{\gamma(0, t ; H, E)} & \quad+\left\|u \mapsto \pi_{2} \mathcal{T}(t-u) \mathcal{B}(Y(u))\right\|_{L^{p}(-1,0 ; \gamma(0, t ; H, E))} \\
& \quad\left\|u \mapsto \pi_{1} \mathcal{T}(t-u) \mathcal{B}(Y(u))\right\|_{L^{2}(0, t ; \gamma(H, E))} \\
& +\left\|u \mapsto \pi_{2} \mathcal{T}(t-u) \mathcal{B}(Y(u))\right\|_{L^{p}\left(-1,0 ; L^{2}(0, t ; \gamma(H, E))\right)}
\end{aligned}
$$

Set $M_{t}:=\sup _{u \in[0, t]}\|\mathcal{T}(u)\|_{\mathcal{L}\left(\mathcal{E}^{p}(E)\right)}$. By the ideal property of the $\gamma$-radonifying operators and the Lipschitz-continuity of $\mathcal{B}$ we have:

$$
\begin{aligned}
\| u & \mapsto \pi_{1} \mathcal{T}(t-u) \mathcal{B}(Y(u)) \|_{L^{2}(0, t ; \gamma(H, E))} \\
& \leq M_{t}\left[t^{\frac{1}{2}}\|B(0)\|_{\gamma(H, E)}+K\|Y\|_{L^{2}\left(0, t ; \mathcal{E}^{p}(E)\right)}\right]
\end{aligned}
$$


where $K$ is the Lipschitz-constant of $B$, and, by equality (17),

$$
\begin{aligned}
\| u & \mapsto \pi_{2} \mathcal{T}(t-u) \mathcal{B}(Y(u)) \|_{L^{p}\left(-1,0 ; L^{2}(0, t ; \gamma(H, E))\right)} \\
& =\left(\int_{-1}^{0}\left\|\pi_{1} \mathcal{T}(t-u+s) \mathcal{B}(Y(u))\right\|_{L^{2}(0, t+s ; \gamma(H, E))}^{p} d s\right)^{\frac{1}{p}} \\
& \leq M_{t}\left[t^{\frac{1}{2}}\|B(0)\|_{\gamma(H, E)}+K\|Y\|_{L^{2}\left(0, t ; \mathcal{E}^{p}(E)\right)}\right] .
\end{aligned}
$$

Process (c) in Theorem 3.2. Note that by Remark 3.3 we may interpret

$$
\int_{0}^{t-u} \mathcal{T}(s) \mathcal{B}(Y(u)) d s
$$

as a $\gamma\left(H, \mathcal{E}^{p}(E)\right)$-valued Bochner integral. To prove that the process

$$
u \mapsto \int_{0}^{t-u} \mathcal{T}(s) \mathcal{B}(Y(u)) d s \in \gamma\left(0, t ; H, \mathcal{E}^{p}(E)\right) \quad \text { a.s. }
$$

observe that by Lemma 2.6 and embedding (16) we have:

$$
\begin{aligned}
\| u & \mapsto \int_{0}^{t-u} \mathcal{T}(s) \mathcal{B}(Y(u)) d s \|_{\gamma\left(0, t ; H, \mathcal{E}^{p}(E)\right)} \\
\lesssim p & \left\|u \pi_{1} \int_{0}^{t-u} \mathcal{T}(s) \mathcal{B}(Y(u)) d s\right\|_{L^{2}(0, t ; \gamma(H, E))} \\
& +\left\|u \mapsto \pi_{2} \int_{0}^{t-u} \mathcal{T}(s) \mathcal{B}(Y(u)) d s\right\|_{L^{p}\left(-1,0 ; L^{2}(0, t ; \gamma(H, E))\right)} .
\end{aligned}
$$

By Minkowski's integral inequality, the ideal property of the $\gamma$-radonifying operators and the Lipschitz-continuity of $\mathcal{B}$ we have:

$$
\begin{aligned}
\| u & \mapsto \pi_{1} \int_{0}^{t-u} \mathcal{T}(s) \mathcal{B}(Y(u)) d s \|_{L^{2}(0, t ; \gamma(H, E))} \\
& \leq t M_{t}\left[t^{\frac{1}{2}}\|B(0)\|_{\gamma(H, E)}+K\|Y\|_{L^{2}\left(0, t ; \mathcal{E}^{p}(E)\right)}\right]
\end{aligned}
$$

and by (17) and Lemma 4.1 we have:

$$
\begin{aligned}
\| u & \mapsto \pi_{2} \int_{0}^{t-u} \mathcal{T}(s) \mathcal{B}(Y(u)) d s \|_{L^{p}\left(-1,0 ; L^{2}(0, t ; \gamma(H, E))\right)} \\
& =\left(\int_{-1}^{0}\left\|u \mapsto \pi_{1} \int_{0}^{t-u+r} \mathcal{T}(s+r) \mathcal{B}(Y(u)) d s\right\|_{L^{2}(0, t ; \gamma(H, E))}^{p} d r\right)^{\frac{1}{p}} \\
& \leq t M_{t}\left[t^{\frac{1}{2}}\|B(0)\|_{\gamma(H, E)}+K\|Y\|_{\left.L^{2}\left(0, t ; \mathcal{E}^{p}(E)\right)\right]}\right.
\end{aligned}
$$

Condition (7) in Theorem 3.2. From the estimates for process (c) above we obtain:

$$
\int_{0}^{t}\|u \mapsto \mathcal{T}(s-u) \mathcal{B}(Y(u))\|_{\gamma\left(0, s ; H, \mathcal{E}^{p}(E)\right)} d s
$$




$$
\lesssim_{p} 2 t M_{t}\left[t^{\frac{1}{2}}\|B(0)\|_{\gamma(H, E)}+K\|Y\|_{L^{2}\left(0, t ; \mathcal{E}^{p}(E)\right)}\right]
$$

Having checked condition (7) and that all processes are in $\gamma(0, t ; H, E)$ a.s. we may apply Theorem 3.2 to obtain the desired result.

Remark 4.3 Let $p^{\prime}$ be such that $\frac{1}{p}+\frac{1}{p^{\prime}}=1$. By testing the stochastic convolution in (18) against elements of $E^{*} \times L^{p^{\prime}}\left(-1,0 ; E^{*}\right)$, which is norming for $\mathcal{E}^{p}(E)$, and applying equality (17) one shows that

$\int_{0}^{t} \pi_{2} \mathcal{T}(t-s) \mathcal{B}(Y(s)) d W_{H}(s)=u \mapsto \int_{0}^{t+u} \pi_{1} \mathcal{T}(t-s+u) \mathcal{B}(Y(s)) d W_{H}(s) \quad$ a.s.

It thus follows from the variation of constants formula (2) that if $Y$ is a generalized strong solution to (SDCP) then $\pi_{2} Y(t)(u)=\pi_{1} Y(t+u)$; in particular it follows that $\pi_{1} Y \in L_{l o c}^{p}(0, \infty ; E)$ a.s.

\subsection{Existence and uniqueness of the solution to (SDCP)}

We continue consider (SDCP) on p. 391. Recall that $\left(\mathcal{F}_{S}\right)_{S \geq 0}$ is a filtration to which $W_{H}$ is adapted. For $t>0, q \in[1, \infty)$ and $r \in[1, \infty]$ let $L_{\mathcal{F}}^{r}\left(0, t ; L^{q}\left(\Omega ; \mathcal{E}^{p}(E)\right)\right)$ be the Banach space of $\left(\mathscr{F}_{S}\right)_{s \geq 0}$ adapted processes in $L^{r}\left(0, t ; L^{q}\left(\Omega ; \mathcal{E}^{p}(E)\right)\right)$. In particular, $L_{\mathcal{F}}^{\infty}\left(0, t ; L^{q}\left(\Omega ; \mathcal{E}^{p}(E)\right)\right)$ is the Banach space of $\left(\mathcal{F}_{S}\right)_{s \geq 0}$ adapted processes $Y$ such that

$$
\|Y\|_{L_{\mathcal{F}}^{\infty}\left(0, t ; L^{q}\left(\Omega ; \mathcal{E}^{p}(E)\right)\right)}=\sup _{0 \leq s \leq t}\left(\mathbb{E}\|Y(s)\|_{\mathcal{E}^{p}(E)}^{q}\right)^{\frac{1}{q}}<\infty
$$

Theorem 4.4 Let the assumptions of Theorem 4.2 hold. In addition, assume that $Y_{0}:=\left[x_{0}, f_{0}\right]^{T} \in L^{q}\left(\mathcal{F}_{0}, \mathcal{E}^{p}(E)\right)$ for some $q \in[2, \infty)$. Then for every $t>0$ and every $r \in[2, \infty]$ there exists a unique process $Y \in L_{\mathcal{F}}^{r}\left(0, t ; L^{q}\left(\Omega ; \mathcal{E}^{p}(E)\right)\right)$ for which (18) holds. In particular, this process is in $L_{\mathcal{F}}^{\infty}\left(0, t ; L^{q}\left(\Omega ; \mathcal{E}^{p}(E)\right)\right)$.

Proof The final remark in the theorem follows from the existence of a solution in $L_{\mathcal{F}}^{\infty}\left(0, t ; L^{q}\left(\Omega ; \mathcal{E}^{p}(E)\right)\right)$ and the uniqueness of the solution in $L_{\mathcal{F}}^{r}\left(0, t ; L^{q}(\Omega\right.$; $\left.\left.\mathcal{E}^{p}(E)\right)\right)$.

Fix $r \in(2, \infty]$ and let $t>0$. Define

$$
L: L_{\mathcal{F}}^{r}\left(0, t ; L^{q}\left(\Omega ; \mathcal{E}^{p}(E)\right)\right) \rightarrow L_{\mathcal{F}}^{r}\left(0, t ; L^{q}\left(\Omega ; \mathcal{E}^{p}(E)\right)\right)
$$

as follows:

$$
L(Z)(s):=\mathcal{T}(s) Y_{0}+\int_{0}^{s} \mathcal{T}(s-u) \mathcal{B}(Z(u)) d W_{H}(u),
$$

where $s \in[0, t]$. Set $M_{t}:=\sup _{0 \leq u \leq t}\|\mathcal{T}(u)\|_{\mathcal{L}\left(\mathcal{E}^{p}(E)\right)}$. To prove that $L(Z)$ is indeed in $L_{\mathcal{F}}^{r}\left(0, t ; L^{q}\left(\Omega ; \mathcal{E}^{p}(E)\right)\right)$, first observe that by inequality (4) and the proof of The- 
orem 4.2, we have:

$$
\begin{aligned}
&(\mathbb{E}\left.\|L(Z)(s)\|_{\mathcal{E}^{p}(E)}^{q}\right)^{\frac{1}{q}} \\
& \lesssim q\left(\mathbb{E}\left\|\mathcal{T}(s) Y_{0}\right\|_{\mathcal{E}^{p}(E)}^{q}\right)^{\frac{1}{q}}+\|u \mapsto \mathcal{T}(s-u) \mathcal{B}(Z(s))\|_{L^{q}\left(\Omega, \gamma\left(0, s ; H, \mathcal{E}^{p}(E)\right)\right)} \\
& \leq M_{t}\left[\left(\mathbb{E}\left\|Y_{0}\right\|_{\mathcal{E}^{p}(E)}^{q}\right)^{\frac{1}{q}}+\left(\mathbb { E } \left[s^{\frac{1}{2}}\|B(0)\|_{\gamma(H, E)}\right.\right.\right. \\
&\left.\left.\left.\quad+K\left(\int_{0}^{s}\|Z(u)\|_{\mathcal{E}^{p}(E)}^{2} d u\right)^{\frac{1}{2}}\right]^{q}\right)^{\frac{1}{q}}\right]
\end{aligned}
$$

and thus from Minkowski's integral inequality, the Hölder inequality and the fact that $r \geq q \geq 2$ we obtain:

$$
\begin{aligned}
(\mathbb{E} & \left.\|L(Z)(s)\|_{\mathcal{E}^{p}(E)}^{q}\right)^{\frac{1}{q}} \\
& \leq M_{t}\left[\left(\mathbb{E}\left\|Y_{0}\right\|_{\mathcal{E}^{p}(E)}^{q}\right)^{\frac{1}{q}}+t^{\frac{1}{2}}\|B(0)\|_{\gamma(H, E)}+K\left(\int_{0}^{s}\left[\mathbb{E}\|Z(u)\|_{\mathcal{E}^{p}(E)}^{q}\right]^{\frac{2}{q}} d u\right)^{\frac{1}{2}}\right] \\
& \leq M_{t}\left[\left(\mathbb{E}\left\|Y_{0}\right\|_{\mathcal{E}^{p}(E)}^{q}\right)^{\frac{1}{q}}+t^{\frac{1}{2}}\|B(0)\|_{\gamma(H, E)}+K s^{\left.\frac{1}{2}-\frac{1}{r}\|Z\|_{L^{r}\left(0, t ; L^{q}\left(\Omega ; \mathcal{E}^{p}(E)\right)\right)}\right]}\right.
\end{aligned}
$$

for every $s \in[0, t]$, where $K$ is the Lipschitz constant of $B$. (In the case $r=\infty$ we interpret $\frac{1}{r}=0$.) Taking $r$ th powers in the above and integrating with respect to $s$ gives that $L(Z) \in L_{\mathcal{F}}^{r}\left(0, t ; L^{q}\left(\Omega ; \mathcal{E}^{p}(E)\right)\right)$. In the same way as the above estimate, one has for $Z_{1}, Z_{2} \in L_{\mathcal{F}}^{r}\left(0, t ; L^{q}\left(\Omega ; \mathcal{E}^{p}(E)\right)\right)$ :

$$
\left\|L\left(Z_{1}\right)-L\left(Z_{2}\right)\right\|_{L_{\mathcal{F}}^{r}\left(0, t ; L^{q}\left(\Omega ; \mathcal{E}^{p}(E)\right)\right)} \lesssim q K t^{\frac{1}{2}} M_{t}\left\|Z_{1}-Z_{2}\right\|_{L_{\mathcal{F}}^{r}\left(0, t ; L^{q}\left(\Omega ; \mathcal{E}^{p}(E)\right)\right)},
$$

so this is a strict contraction for $t$ sufficiently small. Hence by the Banach fixedpoint theorem there exists a unique $Y \in L_{\mathcal{F}}^{r}\left(0, t ; L^{q}\left(\Omega ; \mathcal{E}^{p}(E)\right)\right)$ that satisfies (2). By repeating this argument one obtains a solution for arbitrary $t>0$.

\subsection{Continuity of the Solution to (SDCP)}

Theorem 4.5 Let the assumptions of Theorem 4.2 hold. In addition, assume that $Y_{0}:=\left[x_{0}, f_{0}\right]^{T} \in L^{q}\left(\mathcal{F}_{0}, \mathcal{E}^{p}(E)\right)$ for some $q \in(2, \infty)$. Let $t>0$. Then the solution $Y \in L_{\mathscr{F}}^{\infty}\left(0, t ; L^{q}\left(\Omega ; \mathcal{E}^{p}(E)\right)\right)$ to $(\mathrm{SDCP})$ as given by Theorem 4.4 satisfies $Y \in L^{q}\left(\Omega ; C\left([0, t] ; \mathcal{E}^{p}(E)\right)\right)$.

Proof The statement follows from Corollary 3.5 if it holds that for some $\alpha \in\left(\frac{1}{q}, \frac{1}{2}\right)$ we have:

$$
\sup _{0 \leq s \leq t}\left\|u \mapsto(s-u)^{-\alpha} \mathcal{T}(s-u) \mathcal{B}(Y(u))\right\|_{L^{q}\left(\Omega, \gamma\left(0, s ; \mathcal{E}^{p}(E)\right)\right)}<\infty
$$

Fix $\alpha \in\left(\frac{1}{q}, \frac{1}{2}\right)$ and $s \in[0, t]$. By Lemma 2.6 and embedding (16) we have:

$$
\left\|u \mapsto(s-u)^{-\alpha} \mathcal{T}(s-u) \mathcal{B}(Y(u))\right\|_{\gamma\left(0, s ; \mathcal{E}^{p}(E)\right)}
$$




$$
\begin{aligned}
& \lesssim_{p}\left\|u \mapsto \pi_{1}(s-u)^{-\alpha} \mathcal{T}(s-u) \mathcal{B}(Y(u))\right\|_{L^{2}(0, s ; \gamma(H, E))} \\
& \quad+\left\|u \mapsto(s-u)^{-\alpha} \pi_{2} \mathcal{T}(s-u) \mathcal{B}(Y(u))\right\|_{L^{p}\left(-1,0 ; L^{2}(0, s ; \gamma(H, E))\right)}
\end{aligned}
$$

where $M_{t}:=\sup _{u \in[0, t]}\|\mathcal{T}(u)\|_{\mathcal{L}\left(\mathcal{E}^{p}(E)\right)}$. Concerning the final term in (20); by (17) and by the ideal property of the $\gamma$-radonifying operators we have:

$$
\begin{aligned}
\| u & \mapsto(s-u)^{-\alpha} \pi_{2} \mathcal{T}(s-u) \mathcal{B}(Y(u)) \|_{L^{p}\left(-1,0 ; L^{2}(0, s ; \gamma(H, E))\right)} \\
& =\left[\int_{-1}^{0}\left(\int_{0}^{s+r}(s-u)^{-2 \alpha}\left\|\pi_{1} \mathcal{T}(s-u+r) \mathcal{B}(Y(u))\right\|_{\gamma(H, E)}^{2} d u\right)^{\frac{p}{2}} d r\right]^{\frac{1}{p}} \\
& \leq M_{t}\left[\int_{-1}^{0}\left(\int_{0}^{s}(s-u)^{-2 \alpha}\|B(Y(u))\|_{\gamma(H, E)}^{2} d u\right)^{\frac{p}{2}} d r\right]^{\frac{1}{p}} \\
& =M_{t}\left(\int_{0}^{s}(s-u)^{-2 \alpha}\|B(Y(u))\|_{\gamma(H, E)}^{2} d u\right)^{\frac{1}{2}} .
\end{aligned}
$$

As $q>2$, and using in addition the Lipschitz-continuity of $B$, it follows that:

$$
\begin{aligned}
\| u & \mapsto(s-u)^{-\alpha} \pi_{2} \mathcal{T}(s-u) \mathcal{B}(Y(u)) \|_{L^{q}\left(\Omega ; L^{p}\left(-1,0 ; L^{2}(0, s ; \gamma(H, E))\right)\right)} \\
& \leq M_{t}\left(\int_{0}^{s}(s-u)^{-2 \alpha}\left[\mathbb{E}\|B(Y(u))\|_{\gamma(H, E)}^{q}\right]^{\frac{2}{q}} d u\right)^{\frac{1}{2}} \\
& \leq(1-2 \alpha)^{-\frac{1}{2}} M_{t} s^{\frac{1}{2}-\alpha}\left[\|B(0)\|_{\gamma(H, E)}+K \sup _{u \in[0, s]}\left(\mathbb{E}\|Y(u)\|_{\mathcal{E}^{p}(E)}^{q}\right)^{\frac{1}{q}}\right]<\infty,
\end{aligned}
$$

where $K$ is the Lipschitz constant of $B$. The estimate for the first term on the righthand side of (20) is similar, but slightly simpler; one obtains:

$$
\begin{aligned}
\| u & \mapsto(s-u)^{-\alpha} \pi_{1} \mathcal{T}(s-u) \mathcal{B}(Y(u)) \|_{L^{q}\left(\Omega ; L^{2}(0, s ; \gamma(H, E))\right)} \\
& \leq(1-2 \alpha)^{-\frac{1}{2}} M_{t} s^{\frac{1}{2}-\alpha}\left[\|B(0)\|_{\gamma(H, E)}+K \sup _{u \in[0, s]}\left(\mathbb{E}\|Y(u)\|_{\mathcal{E}^{p}(E)}^{q}\right)^{\frac{1}{q}}\right]<\infty .
\end{aligned}
$$

From the above estimates and the fact that $s^{\frac{1}{2}-\alpha} \leq t^{\frac{1}{2}-\alpha}$ because $\alpha<\frac{1}{2}$, we conclude that (19) holds.

\subsection{Equivalence of solutions to (SDE) and (SDCP)}

Consider the problem (SDE) as given in the introduction with a fixed $p \in[1, \infty)$.

Definition 4.6 A process $X:[-1, \infty) \times \Omega \rightarrow E$ is called a strong solution to (SDE) if it is measurable and adapted to $\left(\mathcal{F}_{t}\right)_{t \geq 0}$ and for all $t \geq 0$ one has:

(i) $\int_{0}^{t}|X(s)|^{2 \vee p} d s<\infty$ a.s.;

(ii) $\left.X\right|_{[-1,0)}=f_{0}$,

(iii) $\int_{0}^{t} X(s) d s \in D(A)$ for all $t>0$ a.s.; 
and

$$
X(t)-x_{0}=A \int_{0}^{t} X(s) d s+C \int_{0}^{t} X_{s} d s+\int_{0}^{t} B\left(X(s), X_{s}\right) d W_{H}(s) \text { a.s. }
$$

Remark 4.7 Note that by condition (i) and Lemma 4.1 one has $\int_{0}^{t} X_{s} d s \in$ $W^{1, p}(-1,0 ; E)$ a.s. Moreover, for any $t>0$; by Minkowski's integral inequality

$$
\begin{aligned}
\left(\int_{0}^{t}\left\|X_{S}\right\|_{L^{p}(E)}^{2} d s\right)^{\frac{1}{2}} & =\left(\int_{0}^{t}\left[\int_{s-1}^{s}\|X(u)\|_{E}^{p} d u\right]^{\frac{2}{p}} d s\right)^{\frac{1}{2}} \\
& \leq\left\|f_{0}\right\|_{L^{p}}+\left(\int_{0}^{t}\left[\int_{0}^{t}\|X(u)\|_{E}^{p \vee 2} d u\right]^{\frac{p \wedge 2}{p \vee 2}} d s\right)^{\frac{1}{p \wedge 2}} \\
& =\left\|f_{0}\right\|_{L^{p}}+t^{\frac{1}{p \wedge 2}}\left[\int_{0}^{t}\|X(u)\|_{E}^{p \vee 2} d u\right]^{\frac{1}{p \vee 2}}<\infty \text { a.s. }
\end{aligned}
$$

Hence by condition (i) the stochastic integral on right hand side of (21) is well defined.

\section{Theorem 4.8}

(i) Let $X$ be a strong solution to (SDE), then the process $Y$ defined by $Y(t):=$ $\left[X(t), X_{t}\right]^{T}$ is a generalized strong solution to (SDCP).

(ii) On the other hand, if $Y$ is a generalized strong solution to (SDCP) then the process defined by $\left.X\right|_{[-1,0)}=f_{0}, X(t):=\pi_{1}(Y(t))$ for $t \geq 0$ is a strong solution to $(\mathrm{SDE})$.

Proof Part (i). In the proof of Theorem 4.2 we saw that $s \mapsto \mathcal{B}(Y(s))$ is stochastically integrable if $Y \in L^{2}\left(0, t ; \mathcal{E}^{p}(E)\right)$ a.s., which follows from Definition 4.6, by Remark 4.7. From Lemma 4.1 above it follows that $Y$ is integrable a.s.:

$$
\int_{0}^{t} Y(s) d s=\left[\begin{array}{c}
\int_{0}^{t} X(s) d s \\
\int_{0}^{t} X_{s} d s
\end{array}\right] \text { a.s. }
$$

and that $\int_{0}^{t} X_{s} d s \in W^{1, p}(-1,0 ; E)$ a.s. and $\int_{0}^{t} X_{s} d s(0)=\int_{0}^{t} X(s) d s \in D(A)$. Hence $\int_{0}^{t} Y(s) d s \in D(\mathcal{A})$ a.s. and again by Lemma 4.1 and by assumption we have, a.s.:

$$
\begin{aligned}
\mathcal{A} \int_{0}^{t} Y(s) d s & =\left[\begin{array}{c}
A \int_{0}^{t} X(s) d s+C \int_{0}^{t} X_{s} d s \\
X_{t}-f_{0}
\end{array}\right] \\
& =\left[\begin{array}{c}
X(t)-x_{0}-\int_{0}^{t} B(X(s)) d W_{H}(s) \\
X_{t}-f_{0}
\end{array}\right] .
\end{aligned}
$$

Combining this equality with the following:

$$
\int_{0}^{t} \mathcal{B}(Y(s)) d W_{H}(s)=\int_{0}^{t}\left[\begin{array}{c}
B\left(Y(s), Y_{s}\right) \\
0
\end{array}\right] d W_{H}(s)=\left[\begin{array}{c}
\int_{0}^{t} B\left(X(s), X_{s}\right) d W_{H}(s) \\
0
\end{array}\right]
$$

we see $Y$ satisfies Definition 3.1. 
Part (ii). Let $Y$ be a generalized strong solution to (SDCP) and define $\left.X\right|_{[-1,0)}=$ $f_{0}, X(t):=\pi_{1}(Y(t))$ for $t \geq 0$. Recall from Remark 4.3 that $\pi_{2} Y(t)=u \mapsto \pi_{1} Y(s+$ $u)=X_{s}$. Thus from the definitions of a generalized strong solution and from the generator $\mathcal{A}$ we obtain

$$
X(s)-x_{0}=A \int_{0}^{t} X(s) d s+C \int_{0}^{t} X_{s} d s+\int_{0}^{t} B\left(X(s), X_{s}\right) d W_{H}(s) \quad \text { a.s. }
$$

Corollary 4.9 $X$ is a strong solution to (SDE) if and only if $X$ satisfies

$$
X(t)=\pi_{1} \mathcal{T}(t)\left[\begin{array}{l}
x_{0} \\
f_{0}
\end{array}\right]+\int_{0}^{t} \pi_{1} \mathcal{T}(t-s) B(X(s)) d W_{H}(s) \quad \text { a.s. }
$$

From Theorem 4.4 and Theorem 4.8 we obtain:

Corollary 4.10 Consider (SDE). Assume $x_{0} \in L^{q}\left(\mathcal{F}_{0} ; E\right)$ and $f_{0} \in L^{q}\left(\mathcal{F}_{0} ; L^{p}\right)$ for some $p \in[1, \infty), q \in[2, \infty)$. Then (SDE) has a unique strong solution in $L^{r}\left(0, t ; L^{q}(\Omega ; E)\right)$ for every $r \in[2, \infty]$ and every $t>0$.

Combining Theorem 4.5 and Theorem 4.8 we obtain:

Corollary 4.11 Consider (SDE). Assume $x_{0} \in L^{q}\left(\mathcal{F}_{0} ; E\right)$ and $f_{0} \in L^{q}\left(\mathcal{F}_{0} ; L^{p}\right)$ for some $p \in[1, \infty), q \in(2, \infty)$. The strong solution $X \in L^{\infty}\left(0, t ; L^{q}(\Omega ; E)\right)$ to $(\mathrm{SDE})$ given by Corollary 4.10 satisfies $X \in L^{q}(\Omega ; C([0, t] ; E))$.

Remark 4.12 One cannot hope to obtain a strong solution to (SDCP) as defined in the monograph of Da Prato and Zabczyk [8], i.e. a process $Y$ such that $Y(t) \in D(\mathcal{A})$ a.s. for all $t \geq 0$ and

$$
Y(t)-\left[\begin{array}{l}
x_{0} \\
f_{0}
\end{array}\right]=\int_{0}^{t} \mathcal{A} Y(s) d s+\int_{0}^{t} \mathcal{B}(Y(s)) d W_{H}(s) \quad \text { a.s. for all } t \geq 0,
$$

unless the problem is deterministic, because of the following:

Proposition 4.13 Let $E=\mathbb{R}$. If a generalized strong solution $Y$ to (SDCP) satisfies $Y(s) \in D(\mathcal{A})$ a.s. for all $s \in[0, t]$ then $\mathcal{T}(s)\left[x_{0}, f_{0}\right]^{T} \in \operatorname{Null}(\mathcal{B})$ and $Y(s)=$ $\mathcal{T}(s)\left[x_{0}, f_{0}\right]^{T}$ a.s. for almost all $s \in[0, t]$, i.e. (SDCP) is deterministic.

Proof Define $X:=\pi_{1}(Y)$, then $X$ is a generalized strong solution to (SDE) by Theorem 4.8. If $Y(s) \in D(\mathcal{A})$ for all $s \in[0, t]$ a.s. then $X \in W^{1, p}(0, t)$ a.s., i.e. by Lemma 4.1 the process $I(\mathcal{B}(Y)):[0, t] \times \Omega \rightarrow \mathbb{R}$ defined by $I(\mathcal{B}(Y))(s)=$ $\int_{0}^{s} \mathcal{B}(Y(u)) d W_{H}(u)$ is in $W^{1, p}(0, t)$ a.s. Recall that the quadratic variation of $I(\mathcal{B}(Y))$ is given by

$$
V_{t}^{2}(I(\mathcal{B}(Y)))=\int_{0}^{t} \mathcal{B}^{2}(Y(s)) d s
$$


and hence by Problem 1.5.11 in [11] the process $I(\mathcal{B}(Y))$ can only be of bounded variation (and hence only possibly in $\left.W^{1, p}(0, t)\right)$ on the set

$$
\begin{aligned}
& \left\{\omega \in \Omega: \int_{0}^{t} \mathcal{B}^{2}(Y(s, \omega)) d s=0\right\} \\
& =\{\omega \in \Omega: Y(s, \omega) \in \operatorname{Null}(\mathcal{B}) \text { for almost all } s \in[0, t]\} .
\end{aligned}
$$

Thus if $I\left(\mathcal{B}^{2}(Y(s))\right)$ is to be in $W^{1, p}(0, t)$ a.s. then one has

$$
Y(s)-\left[\begin{array}{l}
x_{0} \\
f_{0}
\end{array}\right]=\mathcal{A} \int_{0}^{s} Y(u) d u \quad \text { a.s. for all } s \in[0, t],
$$

which implies that $Y(s)=\mathcal{T}(s)\left[x_{0}, f_{0}\right]^{T}$ and $\mathcal{T}(s)\left[x_{0}, f_{0}\right]^{T} \in \operatorname{Null}(\mathcal{B})$ a.s. for all $s \in[0, t]$.

Remark 4.14 We can use Theorem 4.8 to find a stationary solution to (SDE) with additive noise, i.e. $B(Y(s))=b \in \gamma(H, E)$. It follows from Proposition 4.4 in [22] that in this case (SDCP) admits invariant measure if and only if the function

$$
t \mapsto \mathcal{T}(t)[b, 0]^{T}
$$

represents an element of $\gamma\left(0, \infty ; H, \mathcal{E}^{p}(E)\right)$. By Lemma 2.6, embedding (16) and equality (17) this is the case if $\pi_{1} \mathcal{T}(t)[b, 0]^{T} \in L^{2}(0, \infty ; \gamma(H, E))$, i.e. in particular if $(\mathcal{T}(t))_{t \geq 0}$ is exponentially stable.

Acknowledgements The authors wish to thank Anna Chojnowska-Michalik, Jan van Neerven and Mark Veraar for helpful comments.

Open Access This article is distributed under the terms of the Creative Commons Attribution Noncommercial License which permits any noncommercial use, distribution, and reproduction in any medium, provided the original author(s) and source are credited.

\section{References}

1. Albiac, F., Kalton, N.J.: Topics in Banach Space Theory. Graduate Texts in Mathematics, vol. 233. Springer, New York (2006)

2. Bátkai, A., Piazzera, S.: Semigroups for delay equations. Research Notes in Mathematics, vol. 10. AK Peters, Wellesley (2005)

3. Bierkens, J., van Gaans, O., Lunel, S.V.: Existence of an invariant measure for stochastic evolutions driven by an eventually compact semigroup. J. Evol. Equ. 9(4), 771-786 (2009)

4. Brzeźniak, Z.: Stochastic partial differential equations in M-type 2 Banach spaces. Potential Anal. 4(1), 1-45 (1995)

5. Chojnowska-Michalik, A.: Representation theorem for general stochastic delay equations. Bull. Acad. Pol. Sci., Sér. Sci. Math. Astron. Phys. 26(7), 635-642 (1978)

6. Cox, S.G., Veraar, M.C.: Vector-valued decoupling and the Burkholder-Davis-Gundy inequality. Available at http://fa.its.tudelft.nl/ veraar/

7. Crewe, P.: Infinitely delayed stochastic evolution equations in UMD Banach spaces. arXiv:1011. 2615v1

8. Da Prato, G., Zabczyk, J.: Stochastic Equations in Infinite Dimensions. Encyclopedia of Mathematics and Its Applications, vol. 44. Cambridge University Press, Cambridge (1992) 
9. Da Prato, G., Kwapień, S., Zabczyk, J.: Regularity of solutions of linear stochastic equations in Hilbert spaces. Stochastics 23(1), 1-23 (1987)

10. Hoffmann-Jørgensen, J.: Sums of independent Banach space valued random variables. Stud. Math. 52, 159-186 (1974)

11. Karatzas, I., Shreve, S.E.: Brownian Motion and Stochastic Calculus, 2nd edn. Graduate Texts in Mathematics, vol. 113, Springer, New York (1991)

12. Kwapień, S.: On Banach spaces containing $c_{0}$, vol. 52, pp. 187-188. 1974. A supplement to the paper by J. Hoffmann-Jørgensen: "Sums of independent Banach space valued random variables". Stud. Math. 52, 159-186 (1974)

13. Kwapień, S., Woyczyński, W.A.: Tangent sequences of random variables: basic inequalities and their applications. In: Almost Everywhere Convergence, Columbus, OH, 1988, pp. 237-265. Academic Press, Boston (1989)

14. Liu, K.: Stochastic retarded evolution equations: green operators, convolutions, and solutions. Stoch. Anal. Appl. 26(3), 624-650 (2008)

15. Mao, X.: Stochastic Differential Equations and Their Applications. Horwood Publishing Series in Mathematics \& Applications. Horwood, Chichester (1997)

16. Mohammed, S.E.A.: Stochastic Functional Differential Equations. Research Notes in Mathematics, vol. 99. Pitman, Boston (1984) (Advanced Publishing Program)

17. Riedle, M.: Solutions of affine stochastic functional differential equations in the state space. J. Evol. Equ. 8(1), 71-97 (2008)

18. Taniguchi, T., Liu, K., Truman, A.: Existence, uniqueness, and asymptotic behavior of mild solutions to stochastic functional differential equations in Hilbert spaces. J. Differ. Equ. 181(1), 72-91 (2002)

19. van Neerven, J.M.A.M., Riedle, M.: A semigroup approach to stochastic delay equations in spaces of continuous functions. Semigroup Forum 74(2), 227-239 (2007)

20. van Neerven, J.M.A.M., Veraar, M.C.: On the stochastic Fubini theorem in infinite dimensions. In: Stochastic Partial Differential Equations and Applications-VII. Lect. Notes Pure Appl. Math., vol. 245, pp. 323-336. Chapman \& Hall/CRC Press, Boca Raton (2006)

21. van Neerven, J.M.A.M., Weis, L.: Stochastic integration of functions with values in a Banach space. Stud. Math. 166(2), 131-170 (2005)

22. van Neerven, J.M.A.M., Weis, L.: Invariant measures for the linear stochastic Cauchy problem and $R$-boundedness of the resolvent. J. Evol. Equ. 6(2), 205-228 (2006)

23. van Neerven, J.M.A.M., Veraar, M.C., Weis, L.: Stochastic integration in UMD Banach spaces. Ann. Probab. 35(4), 1438-1478 (2007)

24. van Neerven, J.M.A.M., Veraar, M.C., Weis, L.: Stochastic evolution equations in UMD Banach spaces. J. Funct. Anal. 255, 940-993 (2008)

25. Veraar, M., Zimmerschied, J.: Non-autonomous stochastic Cauchy problems in Banach spaces. Stud. Math. 185(1), 1-34 (2008) 